\title{
Termólisis de 4-Hidroxi-3-Metil-2-Butanona y Análisis del Efecto de Solventes a Diferentes Temperaturas
}

\author{
Jennifer J. Lafont ${ }^{(1)^{*}}$, Yudi C. Torres ${ }^{(1)}$ y Edineldo Lans ${ }^{(2)}$ \\ Universidad de Córdoba, (1) Departamento de Química, laboratorio de Cinética y biocombustibles, (2) \\ laboratorio de Química Instrumental, Cra 6 No 76-103, Montería-Colombia. \\ (e-mail: jenniferlafontmendoza@gmail.com; yuditorresvalencia@gmail.com; edilac5201@gmail.com) \\ * autor a quien debe ser dirigida la correspondencia
}

Recibido Nov. 15, 2012; Aceptado Dic. 28, 2012; Versión final recibida Feb. 18, 2013

\section{Resumen}

Se realizó la termólisis del compuesto 4-hidroxi-3-metil-2-butanona solo y en solución con p-xileno, tolueno, benceno y dioxano, a cada 5 grados en el intervalo de temperaturas 463 a $483 \mathrm{~K}$. Se determinaron las constantes de velocidad, el orden de reacción, y los parámetros de activación. Se propone también un mecanismo de termólisis para el compuesto en estudio, y se analiza el efecto del solvente. Las reacciones de termólisis fueron realizadas en un reactor químico pequeño $(150 \mathrm{ml})$ y los productos fueron identificados en un cromatógrafo de gases acoplado a espectrometría de masas. Se encontró que la termólisis del compuesto obedece la ley cinética de primer orden. De acuerdo con sus productos (formaldehido y 2butanona) y a las propiedades fisicoquímicas se propone un estado de transición cíclico de seis miembros concertado y semipolar. Las termólisis de los sistemas en solución presentaron efecto del solvente, los que aceleraron las velocidades de la reacciones.

\section{Thermolysis of 4-hydroxy-3-methyl-2-butanone and analysis of solvent effects at different temperatures}

\begin{abstract}
The thermolysis of the compound 4-hydroxy-3-methyl-2-butanone was carried without solvent and in solution with $p$-xylene, toluene, benzene and dioxane, at every 5 degrees of temperatures in the range 463 to $483 \mathrm{~K}$. The rate constants, the reaction order and the activation parameters were determined. Also, a thermolysis mechanism for the compound under study is proposed and the effect of the solvents is analyzed. Thermolysis was carried out in a small chemical reactor $(150 \mathrm{ml})$ and the products were identified by gas chromatography coupled to mass spectrometry. The results showed that thermolysis of the compound obey a first order kinetics. According to the products (formaldehyde and 2-butanone) and to the physicochemical properties it is proposed a concerted, semi- polar six-member cyclic transition state. The thermolysis in solutions showed solvent effects, which accelerated the reaction rates.
\end{abstract}

Keywords: kinetics, thermolysis, 4-hydroxy-3-methyl-2-butanone, reaction mechanism, solvent effect 


\section{INTRODUCCIÓN}

El compuesto 4-hidroxi-3-metil-2-butanona, es un hidrocarburo alifático de cuatro átomos de carbono, que contiene en su estructura los grupos funcionales cetona e hidroxilo, perteneciente a la familia de las $\beta$ hidroxicetonas, que hasta el momento no ha sido reportada su descomposición térmica, de igual forma se desconoce la influencia que pueda tener el solvente sobre la constante de velocidad en la reacción de termólisis a diferentes temperaturas. Las $\beta$-hidroxicetonas son compuestos químicos de gran importancia en síntesis orgánica debido a que son intermedios de muchas reacciones en la elaboración de medicamentos y otros productos industriales (Kalaitzakis y Smonou, 2010; Padrón et al., 2006). Las $\beta$-hidroxicetonas son obtenidas mediante la dimerización de aldehídos y cetonas en medio ácido o básico bajo ciertas condiciones (reacción aldólica mixta), de tal forma que al aumentar la temperatura pueden ser deshidratados para formar los derivados $\alpha$ - $\beta$-insaturados aldehídicos o cetónicos (Carey y Sundberg, 2007; Bruckner, 2002).

En la literatura científica se han encontrado pocos estudios experimentales sobre la termólisis de compuestos cetónicos con esta característica funcional similar, como es el caso de algunos derivados de la $\beta$-hidroxi pentanona (Yates y Quijano, 1969), en el cual se describe que sus reacciones de descomposición térmica producen cetonas y aldehídos, además de cumplir con la ley cinética de primer orden. Otros estudios describen las termólisis de $\beta$-hidroxi compuestos como: $\beta$-hidroxi-aromáticos (Quijano et al., 2006); $\beta$-hidroxi-nitrilos, (Roux et al., 2007), $\beta$-hidroxi-carboxilatos (Sánchez et al., 2004) y $\beta$-hidroxiésteres; (Zapata et al., 2007; Rodríguez et al., 2009). En este trabajo se evaluó la descomposición térmica del compuesto 4-hidroxi-3-metil-2-butanona en el intervalo de temperaturas entre $(463,15$ y 483,15$) \mathrm{K}$ con variación de cinco grados, para analizar la relación de la constante de velocidad con la temperatura, determinar el orden de reacción, los parámetros de activación y proponer un posible mecanismo de descomposición térmica. De igual forma se evaluó la termólisis del compuesto 4-hidroxi-3-metil-2-butanona en solución con diferentes solventes ( $p$-xileno, tolueno, benceno y dioxano), con el fin de determinar si existe o no una relación isocinética de acuerdo con los postulados de Leffler y Exner, y de esta forma comprobar si hay efecto del solvente en esta serie de reacciones.

El estudio cinético de la termólisis del compuesto 4-hidroxi-3-metil-2-butanona es de gran interés científico, porque brinda información acerca de las velocidades de descomposición térmica del sustrato y de la formación de sus productos a medida que transcurre el tiempo de reacción para cada temperatura estudiada; lo cual permite conocer sus constantes de velocidad, el orden de reacción, los parámetros de activación y proponer un posible mecanismo de descomposición térmica. Así mismo, la importancia de realizar la termólisis de este compuesto en solución, está enfocado en la influencia que puede tener el solvente frente a la velocidad de descomposición del sustrato en estudio; lo cual puede afectar la cinética o el mecanismo de la reacción, debido a las interacciones del solvente con los reactivos o con el complejo activado, ya que este activa las colisiones y/o participa en el rompimiento y formación de los enlaces (March y Smith, 2007); el efecto del solvente puede ser evidenciado con las velocidades de reacción 0 proporciones de los productos (Robson, 2004).

\section{METODOLOGÍA}

Los solventes utilizados en este trabajo ( $p$-xileno, tolueno, benceno y dioxano) fueron de grado analítico con pureza del 99\%, adquiridos de la compañía Merck S.A. El compuesto 4-hidroxi-3-metil-2-butanona (HMB) fue sintetizado en otro trabajo, (Torres, 2012) para ello se utilizó un baño de agua-hielo en el cual se introdujo un balón de tres bocas, en una se le insertó un termómetro, en la segunda un agitador mecánico y por la tercera se adicionó una mezcla de $66,7 \mathrm{~mL}$ de butanona $(0,74$ moles) y $50 \mathrm{~mL}$ de solución fría de formaldehído al 37\% (1,36 moles), el proceso de agitación se realizó durante treinta minutos; luego se adicionó una solución fría de etanol/hidróxido de sodio al 10\% $(2 \mathrm{~mL})$, en dos ocasiones durante la reacción para mantener el pH entre 8 y 9, luego se dejó en reposo por cuatro horas hasta que el olor del formaldehído desapareció, finalmente se neutralizó con ácido acético y se destiló a presión reducida; el rendimiento de la reacción fue del $84 \%$. El producto fue obtenido con pureza del $92 \%$ y determinado cuantitativamente con un equipo de cromatografía de gases acoplado a espectrometría de masas (CG-MS) marca Agilent, modelo 6890N, con detector selectivo de masas marca Agilent, modelo 5973N, se utilizó una columna DB-Wax $(0,25 \mathrm{~mm} \times 30 \mathrm{~m}$ longitud nominal $\times 0,25 \mu \mathrm{m}$ espesor de la película) cuya fase estacionaria fue polietilenglicol y nitrógeno como gas portador.

La termólisis del compuesto 4-hidroxi-3-metil-2-butanona, fue realizada de dos formas diferentes, la primera solo con el reactivo (HMB) (Lafont et al., 2009; Milanés et al., 2012; Ensuncho et al., 2011) y la segunda en solución con distintos solventes: (HMB-p-xileno), (HMB-tolueno), (HMB-benceno) y (HMB-dioxano), (Eyler et al., 1999); todos los sistemas fueron ensayados a las temperaturas de $(463,15 ; 468,15 ; 473,15 ; 478,15$ y $483,15) \mathrm{K}$. El equipo empleado para realizar las termólisis fue un reactor químico de escala semimicro, 
dotado con un sensor de temperatura termocupla tipo K, que puede alcanzar hasta 1073K, un controlador digital Barber-Colman, tipo PID, con incertidumbre de $\pm 1 \mathrm{~K}$; posee además un horno recubierto de asbesto y material refractario que en la parte superior presenta pequeñas perforaciones de $5.5 \mathrm{~cm}$ de diámetro y $8.0 \mathrm{~cm}$ de longitud, donde se introducen las ampolletas de vidrio con la muestra a termolizar.

Para realizar la cinética del compuesto 4-hidroxi-3-metil-2-butanona, se adicionaron con la ayuda de una pipeta transfer $20 \mu \mathrm{L}$ del compuesto $(0.02 \mathrm{ml})$ en cada ampolleta de vidrio cuyas dimensiones eran $2 \mathrm{~mm}$ de diámetro y $4 \mathrm{~cm}$ de longitud, hasta completar seis, posteriormente fueron selladas en ausencia de aire con la llama de un soplete e insertadas en los orificios del reactor previamente calibrado a la temperatura de $463,15 \mathrm{~K}$; luego se fueron sacando las ampolletas del horno a tiempos constantes y se introdujeron en un baño a 273,15K como método físico para frenar la reacción y se procedió a inyectar la muestra en el cromatógrafo de gases acoplada a espectrometría de masas, con el fin de obtener las áreas de descomposición del reactivo y de formación del producto con respecto al tiempo. Este procedimiento se repitió tres veces para cada temperatura estudiada $(463,15 ; 468,15,473,15 ; 478,15$ y 483,15) K (Zapata et al., 2007). Para realizar la termólisis de las soluciones reactivo-solvente fue necesario preparar cuatro soluciones iniciales utilizando $50 \mu \mathrm{L}$ de 4-hidroxi-3-metil-2-butanona y $4950 \mu \mathrm{L}$ del solvente especifico, obteniendo así las muestras a termolizar (HMB-p-xileno), (HMB-tolueno), (HMB-benceno) y (HMB-dioxano), las cuales fueron inyectadas en cada ampolleta con un volumen de $20 \mu \mathrm{L}$ en ausencia de aire y posteriormente selladas hasta completar seis por corrida en forma similar al procedimiento anterior, repitiéndose tres veces para cada solvente a las cinco temperaturas estudiadas $(463,15 ; 468,15,473,15$; $478,15$ y 483,15$) \mathrm{K}$; la concentración de la muestra fue de $5,48 \times 10^{-4} \mathrm{M}$. Las determinaciones cuantitativas de todas las termólisis fueron realizadas utilizando un equipo de cromatografía de gases marca Perkin Elmer, modelo Agilent System XL, con detector FID, se utilizó una columna HP-1 (0,25mm de diámetro interno $\times 30 \mathrm{~m}$ longitud nominal $\times \quad 0,25 \mu \mathrm{m}$ espesor de la película) cuya fase estacionaria fue dimetilpolisiloxano, además se empleó el método cuantitativo del patrón interno (alcohol isopropilico).

Las constantes de velocidad experimentales fueron calculadas siguiendo la ecuación de velocidad de primer orden (1) (Laidler y Meiser, 1998), donde $\lambda_{t}$ representa la disminución de la concentración del reactivo a medida que transcurre el tiempo, $\lambda_{0}$ significa la concentración del reactivo a un tiempo cero, $\mathrm{k}$ es la constante de velocidad y t el tiempo de reacción; los valores de las constantes para cada temperatura de estudio fueron obtenidos graficando el $\operatorname{Ln} \lambda_{t}$ vs $t$ cuya pendiente negativa es igual a la constante de velocidad. Los valores de la energía de activación (Ea) se obtuvieron aplicando la forma logarítmica de la ecuación de Arrhenius (2) mediante el grafico de Ink vs 1/T (K) cuya pendiente de la recta es -Ea/R, siendo $\mathrm{R}$ la constante de los gases; los demás parámetros de activación fueron obtenidos a partir de la ecuación de Eyring (3) que describe el factor pre-exponencial para reacciones unimoleculares en solución, donde $\Delta \mathrm{H}^{\neq}$ es la entalpía de activación, $\mathrm{T}$ la temperatura absoluta $(\mathrm{K}), \mathrm{k}_{\beta}$ es la constante de Boltzman, $\mathrm{h}$ es la constante de Planck y $\Delta \mathrm{S}^{\neq}$es el cambio de entropía en el estado de transición (House, 2007).

$$
\operatorname{Ln} \lambda_{t}=\operatorname{Ln} \lambda_{0}-k t
$$

$\operatorname{Lnk}=-\frac{E_{a}}{R T}+\operatorname{LnA}$

$$
\operatorname{Ln} \frac{k}{T}=\frac{-\Delta H^{\ddagger}}{R} \frac{1}{T}+\operatorname{Ln} \frac{k_{\beta}}{h}+\frac{\Delta S^{\ddagger}}{R}
$$

El efecto del solvente fue verificado de acuerdo con dos postulados diferentes, el primero aplicando la ecuación de Leffler (4) mediante la representación gráfica de $\Delta \mathrm{H}^{\neq}$vs $\Delta \mathrm{S}^{\neq}$para los diferentes sistemas reactivo-solvente ensayados (HMB-p-xileno), (HMB-tolueno), (HMB-benceno) y (HMB-dioxano), cuya linealidad sugiere la existencia de una relación isocinética y la pendiente $(\beta)$ representa la temperatura isocinética (Liu y Guo, 2001; Eyler et al., 1999). En el segundo postulado se analizó el efecto del solvente de acuerdo con un tratamiento estadístico (mínimos cuadrados) más aproximado propuesto por Exner (Berthelot et al., 2001) en el cual se utilizó la ecuación de Arrhenius a través de la representación gráfica de Ink vs $1 / T$ para todos los sistemas reactivo-solvente ensayados; si se observa la convergencia de las líneas en un punto, este corresponde al inverso de la temperatura isocinética $\left(\beta^{-1}\right)$ (Mateo et al., 2004; Cafferata et al., 1991).

$$
\Delta H^{\ddagger}=\Delta H^{0}+\beta \Delta S^{\ddagger}
$$




\section{RESULTADOS Y DISCUSIÓN}

Los productos de las termólisis del compuesto 4-hidroxi-3-metil-2-butanona a las temperaturas de estudio fueron identificados como 2-butanona y formaldehído; por cromatografía de gases acoplada a espectrometría de masas, con un $99 \%$ de correspondencia con las bases de datos de los estándares de referencia NIST disponibles en el equipo; así mismo fueron obtenidos estos productos en las termólisis de los sistemas reactivo-solvente (HMB-p-xileno), (HMB-tolueno), (HMB-benceno) y (HMB-dioxano) en el mismo rango de temperaturas con la concentración inicial de $5,5 \times 10^{-4} \mathrm{M}$ y un $80 \%$ de avance en la reacción de descomposición térmica para cada caso.

Los gráficos de $\operatorname{Ln} \lambda_{\mathrm{t}}$ vs $\mathrm{t}$, representan una serie de datos experimentales que pueden ajustarse a una tendencia lineal con pendiente negativa con $r^{2}$ igual a 0,$9957 ; 0,9983 ; 0,9981 ; 0,9993 ; 0,9983$ para las temperaturas de $(463,15 ; 468,15,473,15 ; 478,15$ y 483,15$) \mathrm{K}$ respectivamente, lo cual comprueba que la reaccion de termólisis para el 4-hidroxi-3-metil-2-butanona sigue una cinética de primer orden; a partir de las pendientes de estos gráficos fueron obtenidos los valores de las constantes de velocidad para cada temperatura, presentadas en la tabla 1; de la cual se puede observar que a medida que aumenta la temperatura se incrementa la velocidad de la reacción, estando en concordancia con la ecuación de Arrhenius, (Carey y Sundberg, 2007), cuyo gráfico es representado en la figura 1 y demuestra esta correlación mediante la tendencia lineal con pendiente negativa cuyo $r^{2}$ es 0,9975 . La relación entre las constantes de velocidad con la temperatura para la reacción unimolecular pueden ser expresadas mediante la ecuación (5).

Tabla 1: Constantes de velocidad del 4-hidroxi-3-metil-2-butanona a diferentes temperaturas

\begin{tabular}{cccc}
\hline $\mathrm{k}\left(\mathrm{s}^{-1}\right)$ & $\mathrm{T}(\mathrm{K})$ & $1000 / \mathrm{T}$ & $\mathrm{Ln} \mathrm{k}$ \\
\hline $7,90 \mathrm{E}-04$ & 463,15 & 2,159 & $-7,14$ \\
1,09E-03 & 468,15 & 2,136 & $-6,82$ \\
$1,57 \mathrm{E}-03$ & 473,15 & 2,113 & $-6,46$ \\
$2,38 \mathrm{E}-03$ & 478,15 & 2,091 & $-6,04$ \\
$3,39 \mathrm{E}-03$ & 483,15 & 2,070 & $-5,69$ \\
\hline
\end{tabular}



Fig. 1: Gráfico de Arrhenius del compuesto 4-hidroxi-3-metil-2-butanona

$\log k=(12,38 \pm 0,07-32,7 \pm 0,3) /(2,302 R T)$

Los parámetros termodinámicos calculados para la reacción de termólisis del compuesto 4-hidroxi-3-metil-2butanona determinados a la temperatura de $473,15 \mathrm{~K}$ son descritos en la tabla 2 , cuyo valor positivo de entalpia de activación $\left(\Delta \mathrm{H}^{\neq}\right)$, puede ser interpretada como un proceso endotérmico, así mismo el valor positivo del cambio de energía libre de activación $\left(\Delta G^{\neq}\right)$indican que la reacción de termólisis no es espontánea, es endergónica, cuya energía interna es insuficiente para que ocurra la degradación térmica, sin embargo el cambio de la entropía de activación $\left(\Delta S^{\ddagger}\right)$ presentó un valor negativo que puede ser 
interpretado como un estado de transición más ordenado, con respecto al reactivo 4-hidroxi-3-metil-2butanona; debido a que posee menos grados de libertad. El valor de la energía de activación (Ea) es cercano al de la entalpia de activación $\left(\Delta \mathrm{H}^{\neq}\right)$, lo cual sugiere que estos valores están acorde con la ecuación $\mathrm{Ea}=\Delta \mathrm{H}^{\neq}+\mathrm{nRT}$; el valor de $\mathrm{A}$, llamado también factor pre-exponencial se encuentra en el intervalo $\left(10^{11}-10^{13} \mathrm{~s}^{-1}\right)$ reportado por la literatura para reacciones de primer orden (Zapata et al., 2007; Julio et al., 2007).

Tabla 2: Parámetros termodinámicos del compuesto 4-hidroxi-3-metil-2-butanona a 473,15K

\begin{tabular}{ccccc}
\hline Ea $\left(\mathrm{kcal} \cdot \mathrm{mol}^{-1}\right)$ & $\log \mathrm{A}\left(\mathrm{s}^{-1}\right)$ & $\Delta \mathrm{H}^{\neq}\left(\mathrm{kcal} \cdot \mathrm{mo}^{-1}\right)$ & $\Delta \mathrm{S}^{\neq}\left(\mathrm{cal} \cdot \mathrm{mol}^{-1} \mathrm{~K}^{-1}\right)$ & $\Delta \mathrm{G}^{\neq}\left(\mathrm{kcal}_{\mathrm{mol}}{ }^{-1}\right)$ \\
\hline $32,7 \pm 0,3$ & $12,38 \pm 0,07$ & $31,8 \pm 0,3$ & $-4,79 \pm 0,05$ & $34,0 \pm 0,9$ \\
\hline
\end{tabular}

Los productos de la termolisis del 4-hidroxi-3-metil-2-butanona obtenidos fueron 2-butanona y formaldehído, en forma similar a las descomposicones termicas realizadas en las $\beta$-hidroxi pentanonas sustituidas con grupos metilo (Yates y Quijano, 1969); de igual forma estuvieron acorde con los valores de los parámetros de activación; lo cual demuestra que los sustituyentes y la longitud de la cadena no influye de manera significativa sobre la velocidad de reacción; de acuerdo con estos productos y valores de activación se propone que el mecanismo de termólisis del 4-hidroxi-3-metil-2-butanona, transcurre a travès de un estado de transición cíclico de seis miembros, semipolar y concertado, el cual produce formaldehído y el enol correspondiente que luego es transformado en 2-butanona por tautomerización; este mecanismo se muestra en la figura 2.<smiles>C/C=C(\C)O</smiles>

Fig. 2: Mecanismo de termólisis del compuesto 4-hidroxi-3-metil-2-butanona

En la tabla 3, se presentan las constantes de velocidad de pseudo primer orden, obtenidas en las reacciones de termólisis del 4-hidroxi-3-metil-2-butanona con los diferentes solventes (HMB- $p$-xileno), (HMB-tolueno), (HMB-benceno) y (HMB-dioxano) a las temperaturas de estudio $(463,15 ; 468,15,473,15$; $478,15$ y 483,15$) \mathrm{K}$, donde se evidencia una tendencia aproximadamente lineal entre las constantes de velocidad y las temperaturas, con valores de $\mathrm{r}^{2}$ entre 0.9994 y 0.9965 , lo cual es un comportamiento acorde con la Ley cinética de primer orden (House, 2007); esto demuestra que los solventes ensayados no participaron en las reacciones de termólisis, ya que se encontraban en exceso con respecto al reactivo, es por ello que el orden de cada descomposición térmica depende solo del 4-hidroxi-3-metil-2-butanona y por lo tanto pueden ser consideradas de pseudo primer orden. Las relaciones entre las constantes de velocidad y las temperaturas están acorde con la expresión de Arrhenius, las cuales pueden ser descritas mediante las ecuaciones 6, 7, 8 y 9 para los sistemas (HMB-p-xileno), (HMB-tolueno), (HMB-benceno) y (HMBdioxano) respectivamente.

$\log k=(11,96 \pm 0,07-31,7 \pm 0,4) /(2,302 R T)$

$\log k=(11,61 \pm 0,04-30,9 \pm 0,7) /(2,302 R T)$ 
$\log k=(10,59 \pm 0,06-28,6 \pm 0,6) /(2,302 R T)$

$\log k=(8,72 \pm 0,03-24,4 \pm 0,3) /(2,302 R T)$

Tabla 3: Constantes de velocidad de pseudo primer orden para las reacciones de termólisis del 4-hidroxi-3-metil-2-butanona con los diferentes solventes

\begin{tabular}{cccc}
\hline Sistema & $\mathrm{T}(\mathrm{K})$ & $\mathrm{k} \times 10^{4}\left(\mathrm{~s}^{-1}\right)$ & $\mathrm{r}^{2}$ \\
\hline \multirow{3}{*}{ HMB-p-xileno } & 463,15 & 8,97 & 0,9985 \\
& 468,15 & 12,8 & 0,9994 \\
& 473,15 & 16,9 & 0,9994 \\
& 478,15 & 24,5 & 0,9993 \\
HMB-tolueno & 483,15 & 38,9 & 0,9985 \\
& 463,15 & 9,92 & 0,9994 \\
& 468,15 & 14,6 & 0,9994 \\
& 473,15 & 19,5 & 0,9983 \\
HMB-benceno & 478,15 & 27,6 & 0,9972 \\
& 483,15 & 40,0 & 0,9974 \\
\hline & 463,15 & 10,9 & 0,9984 \\
& 468,15 & 15,9 & 0,9965 \\
& 473,15 & 21,2 & 0,9974 \\
HMB-dioxano & 478,15 & 28,9 & 0,9993 \\
& 483,15 & 40,6 & 0,9992 \\
\hline & 463,15 & 14,5 & 0,9990 \\
& 468,15 & 19,4 & 0,9984 \\
& 473,15 & 26,2 & 0,9985 \\
& 478,15 & 33,8 & 0,9974 \\
\hline
\end{tabular}

En la tabla 4, se muestran los valores de los parámetros de activación para los sistemas ensayados compuesto-solvente, donde se puede evidenciar que todas las reacciones presentaron datos de energías de activación positivas, las cuales representan la energía mínima requerida que deben alcanzar las moléculas de los reactivos para que colisionen efectivamente y puedan formar el complejo activado; la energía de activación está relacionada en forma inversa con la constante de velocidad de acuerdo con la ecuación de Arrhenius (2), de tal manera que a mayor energía de activación menor es la constante de velocidad y por lo tanto más lenta será la reacción; de tal forma que el sistema HMB-p-xileno presentó mayor valor de Ea por consiguiente su velocidad de reacción fue más lenta que las demás termólisis en solución; en orden ascendente le siguió el HMB-tolueno, HMB-benceno y por último el HMB-dioxano con la reacción más rápida como consecuencia de un dato menor de energía de activación. Si se compara el resultado de la Ea de la termólisis del compuesto 4-hidroxi-3-metil-2-butanona con respecto a los obtenidos en los sistemas con solventes, se encontró mayor valor en el primer caso, lo cual sugiere una menor constante de velocidad y en consecuencia la disminución en la velocidad de la reacción, lo cual significa que la presencia de estos solventes aceleran la velocidad de descomposición térmica.

En cuanto a los valores de los demás parámetros de activación para los sistemas con diferentes solventes, presentaron entalpías de activación $\left(\Delta \mathrm{H}^{\neq}\right)$positivos, al igual que las energías libres de activación $\left(\Delta \mathrm{G}^{\neq}\right)$, pero negativos en las entropías de activación; demostrando procesos de termólisis endotérmicos, no espontáneos, endergónicos y con un estado de transición con menor grados de libertad con respecto al reactivo; en forma general se puede decir que los resultados revelaron un comportamiento muy similar a la termólisis del 4-hidroxi-3-metil-2-butanona sin la presencia de solventes; lo cual sugiere la posibilidad de que su mecanismo también sea semejante. De la tabla 4 también se evidenció la variación en los parámetros de activación con el cambio de los solventes, especialmente entre los valores de entalpías de activación y entropías de activación; se puede apreciar la cercanía entre las entalpias de activación $\left(\Delta \mathrm{H}^{\ddagger}\right)$ de la reacción llevada a cabo en los solventes $p$-xileno, tolueno y benceno y su diferencia con el dioxano, cuyas divergencias son de 7,8; 5,9 y 4,2 respectivamente; de igual forma también existen contrastes entre los datos de las entropías de activación $\left(\Delta S^{\neq}\right)$, de estos tres solventes con respecto al dioxano $(15,96 ; 12,08$ y 8,51 respectivamente) siendo mayor en el p-xileno, le sigue el tolueno y por último el benceno; lo cual indica que existe un efecto del solvente en las termólisis de estos sistemas y que puede estar relacionado con la diferencia en la polaridad de estos solventes; sin embargo los valores de las energías libres de 
activación son muy cercanos entre sí lo cual sugiere que a pesar de que los solventes aceleran la reacción, estas descomposiciones térmicas son equivalentes (Mateo et al., 2004).

Tabla 4: Parámetros de activación de los sistemas con diferentes solventes a 483,15 K

\begin{tabular}{|c|c|c|c|c|}
\hline Sistema & $\mathrm{Ea}\left(\mathrm{kcal} . \mathrm{mol}^{-1}\right)$ & $\Delta \mathrm{H}^{\mp}\left(\mathrm{kcal} \mathrm{mol}{ }^{-1}\right)$ & $\Delta \mathrm{S}^{\neq}\left(\mathrm{cal} \mathrm{mol} \mathrm{mo}^{-1} \mathrm{~K}^{-1}\right)$ & $\Delta \mathrm{G}^{7}\left(\mathrm{kcal} \mathrm{mol}{ }^{-1}\right)$ \\
\hline HMB- $p$-xileno & $31,7 \pm 0,4$ & $31,3 \pm 0,4$ & $-5,50 \pm 0,04$ & $33,9 \pm 0,5$ \\
\hline HMB-tolueno & $30,9 \pm 0,7$ & $29,4 \pm 0,4$ & $-9,38 \pm 0,05$ & $33,8 \pm 0,4$ \\
\hline HMB-benceno & $28,6 \pm 0,6$ & $27,7 \pm 0,7$ & $-12,95 \pm 0,06$ & $33,8 \pm 0,9$ \\
\hline HMB-dioxano & $24,4 \pm 0,3$ & $23,5 \pm 0,3$ & $-21,46 \pm 0,04$ & $33,7 \pm 0,4$ \\
\hline
\end{tabular}

En la figura 3, se observa la representación gráfica del postulado de Leffler para la termólisis de los sistemas en estudio con diferentes solventes, en la cual se comprueba claramente una relación lineal entre las entropías y entalpias de activación acorde con este postulado con una muy buena correlación entre las variables y por lo tanto un excelente efecto de compensación (Liu y Guo, 2001); el valor de la temperatura isocinética obtenida fue de $493,8 \mathrm{~K}$ la cual se encuentra por encima del rango ensayado en las termólisis de los sistemas compuesto-solventes.

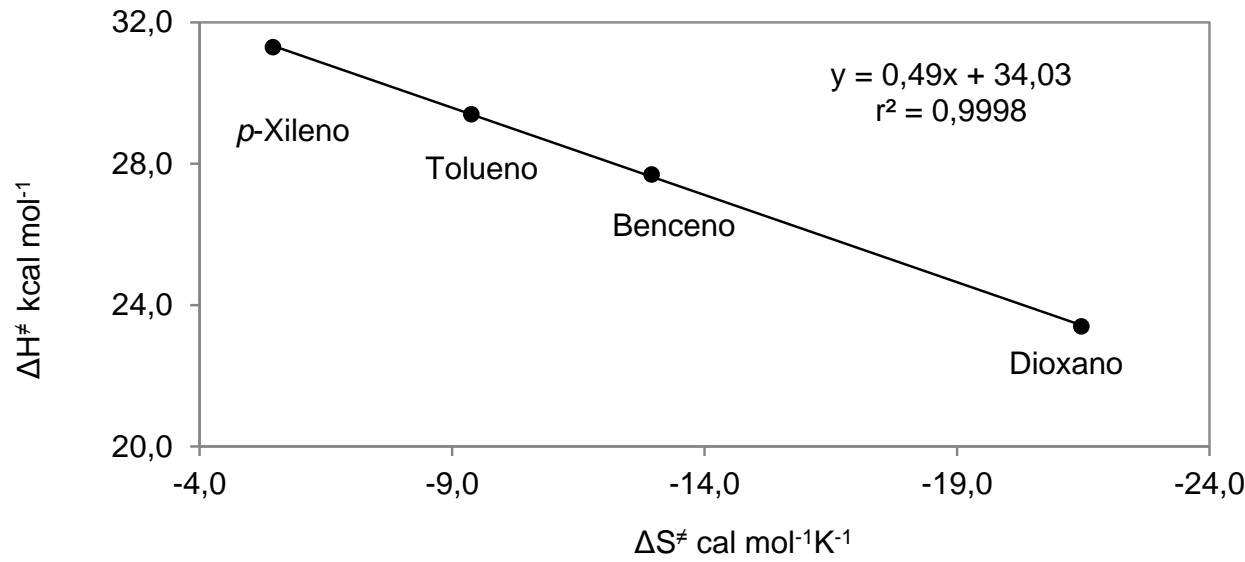

Fig. 3: Relación isocinética de acuerdo con el postulado de Leffler

Debido a que el postulado de Leffler fue cuestionado con el tiempo por estar sujeto a errores, se aplicó un tratamiento estadístico más estricto (libre de errores) para probar la teoría isocinética, basado en los mínimos cuadrados (Nesprias et al., 2007; Cañizo, 2006), el cual fue aplicado para los sistemas en estudio con los diferentes solventes, este modelo propuesto por Exner es representado gráficamente en la figura 4, en la cual se realiza el gráfico de Arrhenius con las regresiones lineales en forma independiente para cada reacción, se comprueba la existencia de una relación isocinética con la convergencia en un punto de intersección entre las líneas, llamada temperatura isocinética, donde todas las reacciones de la serie estudiada deben tener la misma velocidad o equilibrio constante; lo cual representa según este autor una prueba estadística del efecto del solvente en la reacción de termólisis (Cafferata et al., 1991); el valor de la temperatura isocinética o de isoequilibrio calculada a partir del grafico fue de 496,6K.

Se puede decir que existe un efecto de compensación (o compensación entropía- entalpía) porque hay una relación lineal entre las entalpías y entropías de activación (Exner, 2000), cumpliéndose una de las tres características establecidas para este efecto, las otras pueden ser las relaciones entre el logaritmo de los factores preexponencial y las energías de activación, o entre los cambios de entalpía y entropía de una serie de reacciones similares (Liu y Guo, 2001). También se encontró un efecto isocinético o de isoequilibrio, porque se halló la convergencia de las líneas de Arrhenius para cada solvente lo cual indica un comportamiento cinético o termodinámico similar para esta serie de reacciones de termólisis. De acuerdo con lo anterior, las descomposiciones térmicas de los sistemas compuesto-solventes (HMB- $p$-xileno), (HMB-tolueno), (HMB-benceno) y (HMB-dioxano) están acorde con ambos postulados y han demostrado tener una relación isocinética valida, lo cual sugiere una serie de reacciones similares que siguen el mismo mecanismo. 


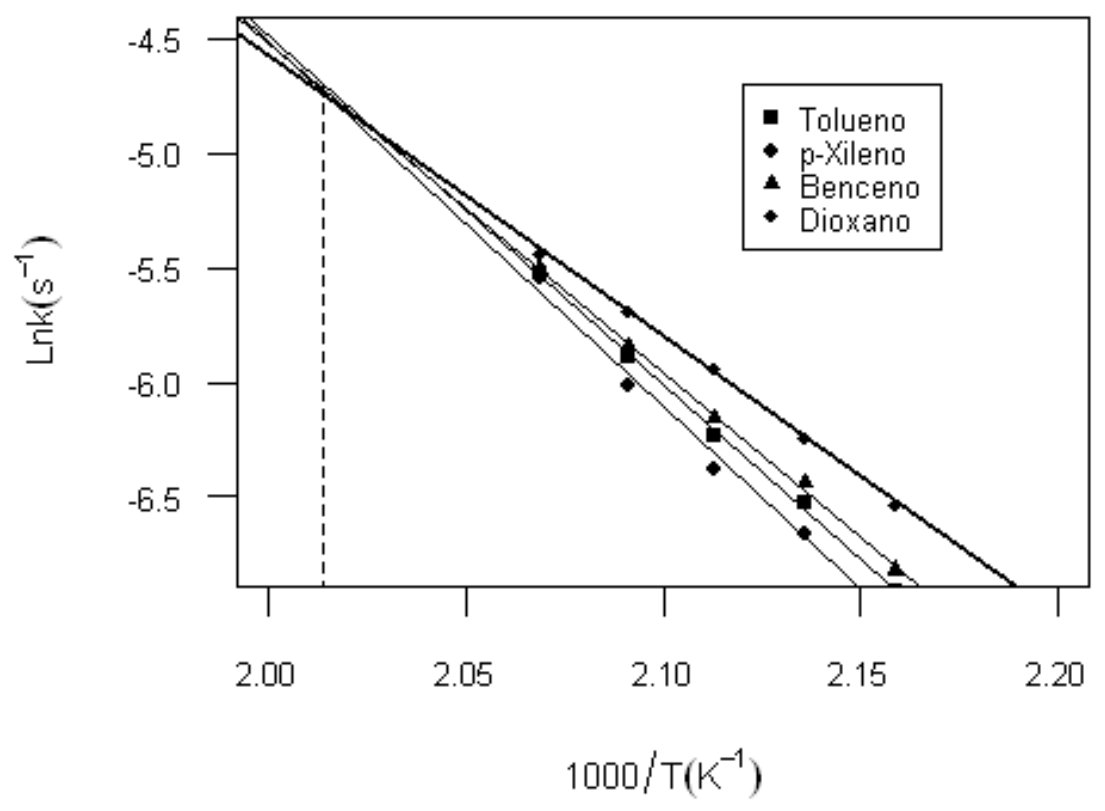

Fig. 4: Gráfico de Arrhenius para los sistemas con diferentes solventes

Se puede decir que existe un efecto de compensación (o compensación entropía- entalpía) porque hay una relación lineal entre las entalpías y entropías de activación (Exner, 2000), cumpliéndose una de las tres características establecidas para este efecto, las otras pueden ser las relaciones entre el logaritmo de los factores preexponencial y las energías de activación, o entre los cambios de entalpía y entropía de una serie de reacciones similares (Liu y Guo, 2001). También se encontró un efecto isocinético o de isoequilibrio, porque se halló la convergencia de las líneas de Arrhenius para cada solvente lo cual indica un comportamiento cinético o termodinámico similar para esta serie de reacciones de termólisis. De acuerdo con lo anterior, las descomposiciones térmicas de los sistemas compuesto-solventes (HMB-tolueno), (HMBp-xileno), (HMB-benceno) y (HMB-dioxano) están acorde con ambos postulados y han demostrado tener una relación isocinética valida, lo cual sugiere una serie de reacciones similares que siguen el mismo mecanismo.

En la figuras 5 se puede observar una tendencia lineal entre el parámetro empírico de polaridad $\mathrm{E}_{\mathrm{T}}(30)$ del solvente y el logaritmo natural de las constantes de velocidad (con $r^{2}=0,9863$ ), que puede interpretarse en términos de la sensibilidad de la reacción en función de la polaridad del solvente; de tal forma la reacción es sensible a cambios de solventes, se hace más rápida frente a solventes mas polares, en este caso el $p$-xileno es el solvente mas polar y por tanto presenta mayor velocidad de la reacción; le sigue el tolueno, benceno y por último el dioxano que es menos polar; estos resultados están acordes a lo encontrado en los parámetros de activación, evidenciándose un efecto del solvente sobre la velocidad de la reacción.

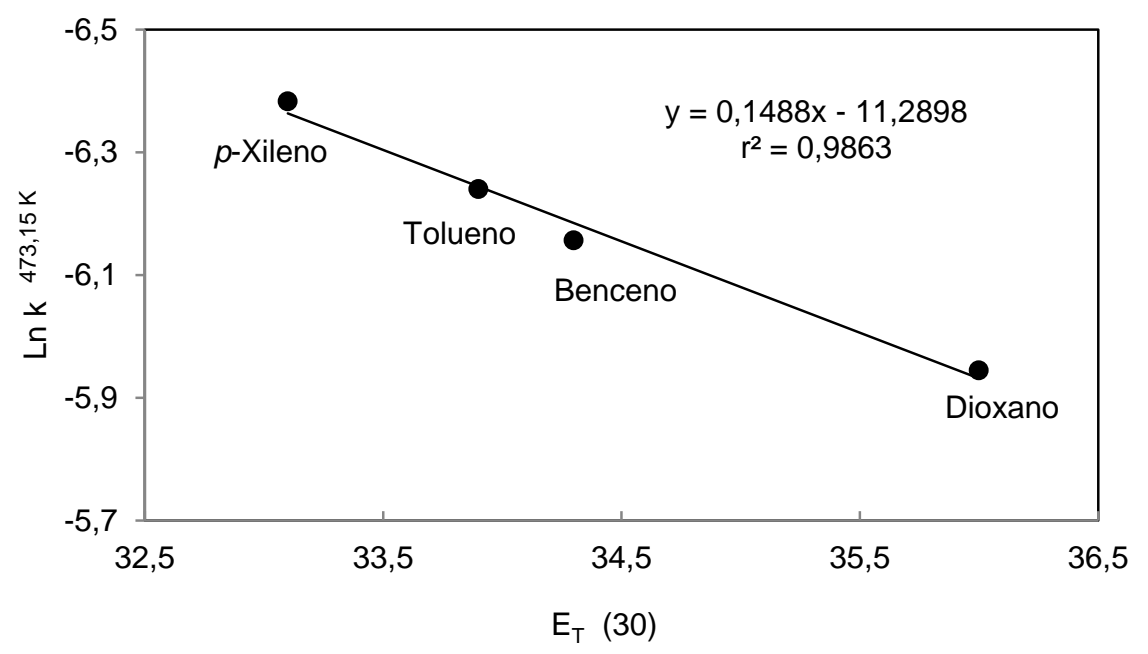

Fig. 5: Efecto de solvente sobre la constante de velocidad a 473,15 K en función del parámetro de polaridad del solvente $\mathrm{E}_{\mathrm{T}}(30)$ 


\section{CONCLUSIONES}

De acuerdo con los resultados obtenidos, su análisis y discusión se puede concluir lo siguiente: 1) La reacción de descomposición térmica del compuesto 4-hidroxi-3-metil-2-butanona realizada en el intervalo de temperaturas entre $(463,15$ y 483,15$) \mathrm{K}$ con cinco grados de diferencia, estuvo acorde con la Ley cinética de primer orden. 2) De acuerdo con los productos obtenidos en la termólisis y con los valores de los parámetros de activación se propone un mecanismo unimolecular con un estado de transición cíclico de seis miembros, concertado y de carácter semipolar. 3) Las reacciones de descomposición térmica de los sistemas en solución (4-hidroxi-3-metil-2-butanona - solvente) siguen una cinética de pseudo primer orden, donde el solvente estuvo en exceso y no influyó en el mecanismo de la reacción. 4) Se comprobó que existe efecto de solventes, los cuales aceleraron la velocidad de las termólisis en solución, siendo mayor con el p-xileno, afectando la cinética de las descomposiciones térmicas.

\section{AGRADECIMIENTOS}

A la Universidad de Córdoba por financiar este proyecto.

\section{REFERENCIAS}

Berthelot, M., Ouvrard, C., T. Lamer., O. Exner, A Program for Linear Regression with a Common Point of Intersection: The Isokinetic Relationship, Journal of Chemical Information and Computer Sciences: 41, 11411144 (2001).

Bruckner, R., Advanced Organic Chemistry Reaction Mechanisms, 5ª edición, 412-420, Elsevier Academic Press, California, EUA (2002).

Cafferata, L. F. y otros cuatro autores, Solvent effect in the thermal decomposition reactions of cyclic ketone diperoxides, Journal Organic Chemistry: 56, 411-414 (1991).

Cañizo, A. I., Cyclic diethylketone triperoxide: preparation, kinetic in solution, solvent effect and its application in polymerization processes, Trends in Organic Chemistry: 11, 55-64 (2006).

Carey, F.A.; R. J. Sundberg, Advanced Organic Chemistry Part A: Structure and Mechanisms, 5a edición, 682-684, Springer Science, New York, EUA (2007).

Ensuncho, A., N. Milanés., J. Robles, Estudio Experimental y Computacional de la Cinética de Termólisis del 2,5-Hexanodiol, Información Tecnológica: 22 (6), 87-94 (2011).

Exner, O., Entropy-enthalpy compensation and anticompensation: solvation and ligand binding, Chemical Communication: 1, 655-1656 (2000).

Eyler, G.N. y otros cuatro autores, Solvent effect in the thermal descomposition reaction of trans-3,3dimethyl-5,6-tetramethylene-1,2,4-trioxacyclohexane; Journal Organic Chemistry: 64, 8457-8460 (1999).

House, J.E., Principles of Chemical Kinetics, 2ª Edición,111-119, Academic Press Elsevier, San Diego, EUA (2007).

Julio, L., J. Lafont., J. Quijano., G. Chuchani, The unimolecular Elimination Kinetics of benzaldoxime in the gas phase, International Journal of Chemical Kinetics: 39, 145-147 (2007).

Kalaitzakis, D., I. Smonou, Highly diastereoselective synthesis of 2-substituted-1,3-diols catalyzed by ketoreductases, Tetrahedron: 66, 9431-9439 (2010).

Lafont, J.J., M. S. Páez., A. Alvis, Estudio Cinético de la Descomposición Térmica de Tres Derivados del Pirano $\left(C_{5} H_{6} O\right)$, Información Tecnológica: 20(5), 39-46 (2009).

Laidler, K.J. y J.H. Meiser, Fisicoquímica, $1^{\text {a }}$ Edición, 353-366. Compañía editorial continental S.A., México D.C, México, (1998).

Liu, L. y Q.X. Guo, Isokinetic Relationship, Isoequilibrium Relationship, and Enthalpy-Entropy Compensation, Chemical Review: 101, 673-695 (2001).

March, J. y M. Smith., Advanced organic chemistry: reactions, mechanisms, and structure, $6^{a}$ edición, 327296, John Wiley \& Sons, Inc, Nueva York, EUA (2007). 
Mateo, C.M., G.N. Eyler., A.I. Cañizo, Efecto de solvente sobre la descomposición térmica de trans-3,6dimetil-3,6-difenil-1,2,4,5-tetraoxaciclohexano en solución, Química Nova: 27 (6), 920-924 (2004).

Milanés, N., A. Ensuncho., J. Robles, Obtención de Dímeros por Termólisis de 1,2-Propanodiol, Información Tecnológica: 23 (5), 3-12 (2012).

Nesprias, K. y otros cinco autores, Preparation and Thermal Decomposition Reaction of p-chloro Benzaldehyde Diperoxide in Tetrahydrofuran Solution, Journal of Energetic Materials: 25 (2), 69-78 (2007).

Padrón, J.M., P.O. Miranda., J.I. Padrón., V.S. Martín, $\beta$-Hydroxy- $\alpha, \beta$-unsaturated ketones: $A$ new pharmacophore for the design of anticancer drugs, Bioorganic \&Medicinal Chemistry Letters: 16(8), 22662269 (2006).

Quijano, J. y otros cuatro autores, Termólisis De B-Hidroxicetonas-B-Aril Sustituidas. Un Estudio Computacional, Dyna: 73 (150), 89-96 (2006).

Robson, M.W., An Introduction to Chemical Kinetics, $1^{\text {a }}$ edición, 263-311, John Wiley \& Sons, Ltd. Chichester, Inglaterra (2004).

Rodriguez, L.D. y otros cinco autores, Theoretical study on the thermolysis of methyl-3-hydroxypropanoate in m-xylene solution, Journal of Molecular Structure: Theochem: 902, 41-48 (2009)

Roux, M. y otros siete autores; Experimental and computational thermochemical study of 3hydroxypropanenitrile, Journal of Chemical Thermodynamics: 39 (10), 1377-1383 (2007).

Sánchez, C., J. Quijano., R. Notario, Theoretical study of the thermolysis reaction of methyl $\beta$ hydroxycarboxylates in the gas phase, Journal Physical Organic Chemistry: 17(4), 294 - 302 (2004).

Torres, Y., Termólisis de 4-hidroxi-3-metil-2-butanona y efecto del solvente, Tesis de Magíster, Departamento de Química, Universidad de Córdoba, Montería, Colombia (2012).

Yates, B.L. y J. Quijano, The Thermal Decomposition of $\beta$-Hydroxy Ketones, The Journal of Organic Chemistry: 34(9), 2506-2508 (1969).

Zapata, E., J. Gaviria., J. Quijano, Thermal descomposition of methyl $\beta$-Hydroxyesters in $m$-xylene solution, International Journal of Chemical kinetics: 39 (2), 92-96 (2007). 